\title{
Correction to: Compositional dynamics of suspended sediment in the Rhine River: sources and controls
}

\author{
Marcel van der Perk ${ }^{1}$ - Alvaro Espinoza Vilches ${ }^{1}$ \\ Published online: 13 March 2020 \\ (C) Springer-Verlag GmbH Germany, part of Springer Nature 2020
}

Erratum to: Journal of Soils and Sediments (2020) 20:1754-1770 https://doi.org/10.1007/s11368-019-02490-5

Table 4 in the originally published article is not correct.

Correct Table 4 is shown below.

The online version of the original article can be found at https://doi.org/ 10.1007/s11368-019-02490-5

Marcel van der Perk

m.vanderperk@uu.nl

1 Department of Physical Geography, Utrecht University,

P.O. Box 80.115, 3508, TC Utrecht, the Netherlands 
Table 4 Results of PCA of element concentrations in suspended sediment in the Rhine River.

a. Not-normalised concentrations

\begin{tabular}{|c|c|c|c|c|c|}
\hline & Component 1 & Component 2 & Component 3 & Component 4 & Component 5 \\
\hline $\begin{array}{l}\text { Proportion of variance } \\
\text { explained }\end{array}$ & 0.460 & 0.234 & 0.059 & 0.046 & 0.024 \\
\hline \multicolumn{6}{|c|}{ Elements with positive loadings } \\
\hline Values $>0.20$ & - & $\begin{array}{l}\mathrm{Ba}, \mathrm{Ti}, \mathrm{Cr}, \mathrm{Cu} \text {, } \\
\mathrm{Ag}, \mathrm{Zn}, \mathrm{Cd}, \mathrm{Hg} \text {, } \\
\mathrm{Sn}, \mathrm{Pb}, \mathrm{As} \text {, and } \\
\mathrm{Sb}\end{array}$ & $\mathrm{Nb}(+0.36)$ & $\begin{array}{l}\mathrm{K}, \mathrm{Nb}, \mathrm{Mo} \\
\mathrm{Mn}, \mathrm{P}(+0.47), \\
\mathrm{Sb}\end{array}$ & $\begin{array}{l}\mathrm{Sm}(+0.54), \mathrm{W} \\
\mathrm{Mn}, \mathrm{Co}, \mathrm{Ni}\end{array}$ \\
\hline $\begin{array}{l}\text { Values between } \\
0.15 \text { and } 0.20\end{array}$ & - & $\begin{array}{l}\mathrm{Na}, \mathrm{La}, \mathrm{W}, \mathrm{Mn} \text {, } \\
\text { and Te }\end{array}$ & Co & - & Mo \\
\hline
\end{tabular}

\begin{tabular}{|c|c|c|c|c|c|}
\hline \multicolumn{6}{|c|}{ Elements with negative loadings } \\
\hline Values $<-0.20$ & - & - & $\begin{array}{l}\mathrm{Na}, \mathrm{K}, \mathrm{Sm}, \\
\mathrm{Zr}(-0.39), \mathrm{W}, \\
\text { and Sn }(-0.30)\end{array}$ & $\begin{array}{l}\mathrm{Mg}, \mathrm{Ca}(-0.37) \text {, } \\
\text { and } \mathrm{Sr}(-0.31)\end{array}$ & $\mathrm{K}, \mathrm{Ti}$, and $\mathrm{Nb}$ \\
\hline $\begin{array}{l}\text { Values between } \\
-0.15 \text { and }-0.20\end{array}$ & $\begin{array}{l}\mathrm{Li}, \mathrm{K}, \mathrm{Rb}, \mathrm{Cs} \text {, } \\
\mathrm{Be}, \mathrm{REEs} \\
\text { (except La and } \\
\mathrm{Sm}), \mathrm{Fe}, \mathrm{Co}, \mathrm{Ni} \text {, } \\
\mathrm{Al}, \mathrm{Ga}, \mathrm{Tl}\end{array}$ & - & & & $\begin{array}{l}\mathrm{Rb}, \mathrm{V}, \mathrm{Tl} \text {, and } \\
\mathrm{Sb}\end{array}$ \\
\hline
\end{tabular}

Relations with component scores

Q

Clay $(<2 \mu \mathrm{m})$

Org. C $\begin{array}{ll}r=-0.48 ; & r=-0.59 ; \\ p<0.001 & p<0.001\end{array}$

$$
p<0.001
$$

\begin{tabular}{cl}
$\mathbf{Q}$ & $\begin{array}{l}r=-0.48 ; \\
p<0.001\end{array}$ \\
& \\
Clay $(<2 \mu \mathrm{m})$ & $r=-0.63 ;$ \\
& $p<0.001$ \\
Org. $C$ & $r=0.61 ; p<$ \\
& 0.001 \\
\hline Remarkable trends in component scores
\end{tabular}

Long-term trend

Seasonality of monthly averaged scores

-
Strong
seasonality
with negative
values
between
October and
March and
positive values
between April
and
September
with a peak in
May

Jumpwise

increase in

early 2013

Weak, but

clear seasonal pattern with generally negative values from January through August and positive values from September through December

$\begin{aligned} & \text { n.s. }(p>0.10), \\ & \text { but scores } \\ & \text { seem to show } \\ & \text { a delayed } \\ & \text { negative } \\ & \text { response }\end{aligned}$
$\begin{array}{lll}\text { n.s. }(p>0.10) & \quad r=0.21 ; \\ & p=0.008 & \text { n.s. }(p>0.10) \\ \text { n.s. }(p>0.10) & r=0.68 ; \\ & p<<0.001 & \text { n.s. }(p>0.10)\end{array}$

n.s. $(p>0.10)$ 
Table 4 (continued)

b. Al-normalised concentrations

\begin{tabular}{|c|c|c|c|c|c|}
\hline & Component 1 & Component 2 & Component 3 & Component 4 & Component 5 \\
\hline $\begin{array}{l}\text { Proportion of variance } \\
\text { explained }\end{array}$ & 0.523 & 0.137 & 0.058 & 0.041 & 0.035 \\
\hline \multicolumn{6}{|c|}{ Elements with positive loadings } \\
\hline Values $>0.20$ & - & $\mathrm{Zr}$ and $\mathrm{Sn}$ & $\begin{array}{l}\mathrm{Li}, \mathrm{K}(0.37), \mathrm{Rb} \text {, } \\
\mathrm{Mg}, \mathrm{Ca}, \mathrm{Sm} \text {, } \\
\text { and P }\end{array}$ & $\begin{array}{l}\mathrm{Ca}, \mathrm{Sr}, \mathrm{Nb} \\
(0.31) \text {, and } \mathrm{Mn}\end{array}$ & $\begin{array}{l}\mathrm{Mg}(0.33), \mathrm{Ca} \text {, } \\
\mathrm{Sr}, \mathrm{U}\end{array}$ \\
\hline $\begin{array}{l}\text { Values between } \\
0.15 \text { and } 0.20\end{array}$ & - & $\begin{array}{l}\mathrm{Ba}, \mathrm{W}, \mathrm{Sb} \text {, and } \\
\mathrm{Te}\end{array}$ & Sr, Th, and W & $\begin{array}{l}\mathrm{Mg}, \mathrm{La}, \mathrm{P} \text {, and } \\
\mathrm{Sb}\end{array}$ & $\mathrm{K}, \mathrm{Hg}$, and $\mathrm{Ga}$ \\
\hline \multicolumn{6}{|c|}{ Elements with negative loadings } \\
\hline Values $<-0.20$ & - & - & - & $\begin{array}{l}\mathrm{Li}, \mathrm{Ce}, \mathrm{Pr}, \mathrm{Tl} \\
\text { and } \mathrm{Zr}\end{array}$ & $\begin{array}{l}\text { Nb, Mo, Ge, P } \\
(-0.32) \text {, and Sb }\end{array}$ \\
\hline $\begin{array}{l}\text { Values between } \\
-0.15 \text { and }-0.20\end{array}$ & $\begin{array}{l}\text { majority of } \\
\text { metals }\end{array}$ & $\begin{array}{l}\text { REEs except } \\
\text { La, Pr, and Sm }\end{array}$ & $\begin{array}{l}\text { Contaminant } \\
\text { metals } \mathrm{Cr}, \mathrm{Cu} \text {, } \\
\mathrm{Ag}, \mathrm{Zn}, \mathrm{Cd}, \mathrm{Hg} \text {, } \\
\mathrm{Pb}\end{array}$ & $\mathrm{Nd}, \mathrm{Th}$, and $\mathrm{Sn}$ & $\begin{array}{l}\mathrm{K}, \mathrm{Be}, \mathrm{Ce} \text {, and } \\
\mathrm{Pr}\end{array}$ \\
\hline \multicolumn{6}{|c|}{ Relations with component scores } \\
\hline $\mathbf{Q}$ & $\begin{array}{l}r=0.60 \\
p<<0.001\end{array}$ & $\begin{array}{l}r=-0.33 \\
p<<-0.001\end{array}$ & n.s. $(p>0.10)$ & $\begin{array}{l}r=-0.21 \\
p=0.009\end{array}$ & $\begin{array}{l}r=-0.23 \\
p=0.004\end{array}$ \\
\hline Clay $(<2 \mu \mathrm{m})$ & $\begin{array}{l}r=0.49 \\
p<<0.001\end{array}$ & $\begin{array}{l}r=-0.23 \\
p=0.004\end{array}$ & n.s. $(p>0.10)$ & $\begin{array}{l}r=-0.31 \\
p<00.001\end{array}$ & n.s. $(p>0.10)$ \\
\hline Org. C & $\begin{array}{l}r=-0.44 \\
p<<0.001\end{array}$ & $\begin{array}{l}r=0.30 \\
p<<0.001\end{array}$ & $\begin{array}{l}r=0.31 \\
p<00.001\end{array}$ & $\begin{array}{l}r=0.26 \\
p=0.001\end{array}$ & $\begin{array}{l}r=-0.58 \\
p<<0.001\end{array}$ \\
\hline
\end{tabular}

Remarkable trends in component scores

Long-term trend

Seasonality of monthly averaged scores

\begin{tabular}{|c|c|c|c|}
\hline $\begin{array}{l}\text { Jumpwise } \\
\text { decrease in } \\
\text { early } 2013\end{array}$ & $\begin{array}{l}\text { Slightly } \\
\text { decreasing } \\
\text { trend }\end{array}$ & $\begin{array}{l}\text { Slight } \\
\text { jumpwise } \\
\text { increase in } \\
\text { April } 2014\end{array}$ & - \\
\hline - & $\begin{array}{l}\text { Weak but clear } \\
\text { seasonal trend } \\
\text { with positive } \\
\text { values from } \\
\text { March to } \\
\text { August and } \\
\text { negative } \\
\text { values from } \\
\text { September to } \\
\text { February }\end{array}$ & $\begin{array}{l}\text { Weak but clear } \\
\text { seasonal trend } \\
\text { with positive } \\
\text { values from } \\
\text { April to August } \\
\text { and generally } \\
\text { negative } \\
\text { values from } \\
\text { September to } \\
\text { March }\end{array}$ & $\begin{array}{l}\text { Weak but clear } \\
\text { seasonal trend } \\
\text { with negative } \\
\text { values from } \\
\text { January } \\
\text { through June } \\
\text { and positive } \\
\text { values during } \\
\text { the second } \\
\text { half of the } \\
\text { year }\end{array}$ \\
\hline
\end{tabular}


Table 4 (continued)

c. Organic-C-normalised concentrations

\begin{tabular}{|c|c|c|c|c|c|}
\hline & Component 1 & Component 2 & Component 3 & Component 4 & Component 5 \\
\hline $\begin{array}{l}\text { Proportion of variance } \\
\text { explained }\end{array}$ & 0.440 & 0.244 & 0.055 & 0.063 & 0.044 \\
\hline \multicolumn{6}{|c|}{ Elements with positive loadings } \\
\hline Values $>0.20$ & - & $\begin{array}{l}\mathrm{Ba}, \mathrm{Ti}, \mathrm{V}, \mathrm{Cr}, \\
\mathrm{Cu}, \mathrm{Ag}, \mathrm{Zn}, \mathrm{Cd} \text {, } \\
\mathrm{Hg}, \mathrm{Sn}, \mathrm{Pb}, \mathrm{As}, \\
\text { and } \mathrm{Sb}\end{array}$ & $\mathrm{Nb}$ & $\begin{array}{l}\mathrm{Nb}, \mathrm{Mo} \text {, } \\
\mathrm{Mn}(0.33) \text {, } \\
\mathrm{P}(0.48) \text {, and } \\
\mathrm{Sb}\end{array}$ & $\begin{array}{l}\mathrm{Sm}(0.44), \mathrm{W} \text {, } \\
\mathrm{Ni} \text {, and } \mathrm{Ge}\end{array}$ \\
\hline $\begin{array}{l}\text { Values between } \\
0.15 \text { and } 0.20\end{array}$ & $\mathrm{Ca}$ & $\begin{array}{l}\mathrm{Na}, \mathrm{La}, \mathrm{W}, \mathrm{Mn} \text {, } \\
\text { and Te }\end{array}$ & Co & K & Co \\
\hline
\end{tabular}

\begin{tabular}{|c|c|c|c|c|c|}
\hline \multicolumn{6}{|l|}{ Elements with negative loadings } \\
\hline Values $<-0.20$ & - & - & $\begin{array}{l}\mathrm{Na}, \mathrm{K}(-0.33) \text {, } \\
\mathrm{Sm}, \mathrm{Zr}(-0.39) \text {, } \\
\text { and } \mathrm{Sn}\end{array}$ & $\mathrm{Ca}$ & $\begin{array}{l}\mathrm{K}, \mathrm{Rb}, \mathrm{La}, \mathrm{Ti} \text {, } \\
\text { and } \mathrm{Nb}\end{array}$ \\
\hline $\begin{array}{l}\text { Values between } \\
-0.15 \text { and }-0.20\end{array}$ & $\begin{array}{l}\mathrm{Li}, \mathrm{Rb}, \mathrm{Cs}, \mathrm{Be} \text {, } \\
\mathrm{Y}, \text { majority of } \\
\text { REEs (except } \\
\mathrm{La} \text { and } \mathrm{Sm}), \mathrm{V} \text {, } \\
\mathrm{Fe}, \mathrm{Al} \text {, and Ga }\end{array}$ & - & $\mathrm{Li}, \mathrm{W}$, and $\mathrm{Te}$ & $\begin{array}{l}\mathrm{Mg}, \mathrm{Sr}, \mathrm{U}, \mathrm{Cr} \text {, } \\
\text { and } \mathrm{Hg}\end{array}$ & $\mathrm{V}, \mathrm{Al}$, and $\mathrm{Sb}$ \\
\hline \multicolumn{6}{|c|}{ Relations with component scores } \\
\hline $\mathbf{Q}$ & $\begin{array}{l}r=-0.48 \\
(p<00.001)\end{array}$ & $\begin{array}{l}r=-0.59 \\
(p<<0.001)\end{array}$ & $\begin{array}{l}\text { n.s. } \\
(p>0.10)\end{array}$ & $\begin{array}{l}\text { n.s. } \\
(p>0.10)\end{array}$ & $\begin{array}{l}r=0.18 \\
(p=0.02)\end{array}$ \\
\hline Clay $(<2 \mu \mathrm{m})$ & $\begin{array}{l}r=-0.65 \\
(p<<0.001)\end{array}$ & $\begin{array}{l}r=-0.26 \\
(p<0.001)\end{array}$ & $\begin{array}{l}\text { n.s. } \\
(p>0.10)\end{array}$ & $\begin{array}{l}\text { n.s. } \\
(p>0.10)\end{array}$ & $\begin{array}{l}\text { n.s. } \\
(p>0.10)\end{array}$ \\
\hline Org. C & $\begin{array}{l}r=0.41 ; \\
(p<<0.001)\end{array}$ & $\begin{array}{l}r=0.28 \\
(p<0.001)\end{array}$ & $\begin{array}{l}\text { n.s. } \\
(p>0.10)\end{array}$ & $\begin{array}{l}r=0.71 \\
(p<<0.001)\end{array}$ & $\begin{array}{l}\text { n.s. } \\
(p>0.10)\end{array}$ \\
\hline \multicolumn{6}{|c|}{ Remarkable trends in component scores } \\
\hline Long-term trend & - & - & $\begin{array}{l}\text { Increase in } \\
\text { second half } \\
2012 \text { / early } \\
2013\end{array}$ & - & $\begin{array}{l}\text { Jumpwise } \\
\text { decrease in } \\
\text { April } 2014\end{array}$ \\
\hline $\begin{array}{l}\text { Seasonality of monthly } \\
\text { averaged scores }\end{array}$ & $\begin{array}{l}\text { Strong } \\
\text { seasonality } \\
\text { with negative } \\
\text { values } \\
\text { between } \\
\text { November and } \\
\text { March and } \\
\text { positive values } \\
\text { between April } \\
\text { and October }\end{array}$ & - & - & $\begin{array}{l}\text { Weak but clear } \\
\text { seasonal trend } \\
\text { with positive } \\
\text { values from } \\
\text { February to } \\
\text { June and } \\
\text { negative } \\
\text { values during } \\
\text { the rest of the } \\
\text { year }\end{array}$ & - \\
\hline
\end{tabular}

